\title{
Relationship Between Aortic Calcification and Stroke in a Mass Screening Program Using a Mobile Helical Computed Tomography Unit
}

\author{
Yasutaka Itani, MD; Shigeru Watanabe, MD; Yoshiaki Masuda, MD
}

\begin{abstract}
Background It is reported that there is a significant relationship between the calcification of the aortic arch detected by chest X-ray examination and stroke. However, the relationship between stroke and aortic calcification $(\mathrm{AoC})$ detected during a mass screening using a mobile helical computed tomography $(\mathrm{CT})$ unit remains unknown.

Methods and Results The study population consisted of 2,618 subjects (1,345 men, and 1,273 women; mean age, $52.9 \pm 13.8$ years) who participated in a mass CT screening for lung cancer and tuberculosis. In the present study, 28 subjects ( 18 men, and 10 women; mean age, $65.9 \pm 13.5$ years) had a past history of cerebral infarction. There were no subjects with a past history of intracerebral or subarachnoid hemorrhage. The frequency of AoC was significantly higher in the infarction group who were older than 50 years of age. In logistic regression analysis, the AoC was a stronger contributor of infarction than sex, age, and risk factors. Furthermore, the odds ratio of AoC for subjects with a past history of infarction increased as the number of calcified segments increased, and these values were 1.82 (95\% confidence interval (CI) 1.06-3.15) in men, and 2.53 (95\% CI 1.12-5.75) in women. Conclusions These results suggest that detection of AoC during mass chest screening using a mobile helical CT unit is an effective way to evaluate the risk of cerebral infarction. (Circ J 2006; 70: 733-736)
\end{abstract}

Key Words: Aortic calcification; Cerebral infarction; Mass screening; Mobile helical CT

$\mathbf{T}$ he development of computed tomography (CT) has contributed to the noninvasive imaging of cardiovascular organs. Previous studies suggest that coronary artery disease (CAD) is significantly related to coronary artery calcification $(\mathrm{CAC})^{1-8}$ and aortic calcification $(\mathrm{AoC})^{9,10}$ detected by $\mathrm{CT}$, and that $\mathrm{CAC}$ is predictive of the future onset of CAD and cardiovascular death!1-15 Stenosis, occlusion, and vulnerable plaque in the coronary artery can now be detected using multidetector-row CT (MDCT) ${ }^{16,17}$

In 1994, a mobile helical CT unit was developed in Japan, which enabled large-scale medical examinations to be conducted wherever they are needed. Since 1995, this CT unit has been used for a mass chest screening program for lung cancer ${ }^{18,19}$ In the current CT screening program, it is effective in diagnosing not only respiratory diseases by using the lung display, but also morphological abnormalities in the cardiovascular system by using the mediastinum display. Therefore, we have previously investigated frequencies of $\mathrm{CAC}$ and $\mathrm{AoC}$, and their relationship to a past history of CAD (myocardial infarction, coronary angioplasty, and coronary artery bypass grafting) ${ }_{2}^{20,21}$ the standard values of aortic diameters and the frequency of aortic aneurysm ${ }^{22}$ and the relationship between CAC and death caused by cardiac disease in a 4-year follow up ${ }^{23}$ in the general population who had participated in this CT screening program.

As a result of our investigations, the odds ratio for

(Received December 13, 2005; revised manuscript received March 1, 2006; accepted March 17, 2006)

The Japanese Society of CT Screening, Tokyo, Japan

Mailing address: Yasutaka Itani, MD, 5-11-1 Shishibone, Edogawa-

ku, Tokyo 133-0073, Japan. E-mail: itani-y@mtg.biglobe.ne.jp persons with a past history of CAD was the highest in men under 60 years of age with $\mathrm{CAC}^{20}$ and tended to increase as the number of calcified aortic segments increased ${ }^{21}$ Aortic diameters were found to increase with age, and 11 cases with asymptomatic aortic aneurysm were detected 22 Furthermore, the frequency of CAC was higher in the group with cardiac death than that in the non-cardiac death group ${ }^{23}$ In contrast, several previous studies have reported that CAC detected by an electron-beam CT (EBCT) ${ }^{24}$ and calcification in the aortic arch (Arch) detected on plain chest $\mathrm{X}$-rays,, 25 are significantly related to stroke.

However, the relationship between stroke and $\mathrm{AoC}$ detected by $\mathrm{CT}$ in the general population remains unknown. Therefore, in the present study, we investigated the relationship between $\mathrm{AoC}$ and stroke in the population who participated in a mass chest screening program for lung cancer and tuberculosis using a mobile helical CT unit.

\section{Methods}

A total of 2,618 subjects (1,345 men, 1,273 women; age range, $15-90$ years; mean age, $52.9 \pm 13.8$ years) were invited to participate in a chest examination program that used a mobile helical CT unit to screen for lung cancer and tuberculosis. All medical examinations were performed by the Chiba Anti-Tuberculosis Association between 1995 and 1999, after all participants gave informed consent. The mobile helical CT unit consisted of a remodeled bus with a built-in helical CT scanner (CT-W950SR; Hitachi Medical Corporation, Tokyo, Japan).

CT scanning was performed with a tube voltage of $120 \mathrm{kV}$ and a tube current of $50 \mathrm{~mA}$, with $10 \mathrm{~mm}$-thick consecutive sections, $10 \mathrm{~mm} / \mathrm{s}$ table speed and shallow breath- 


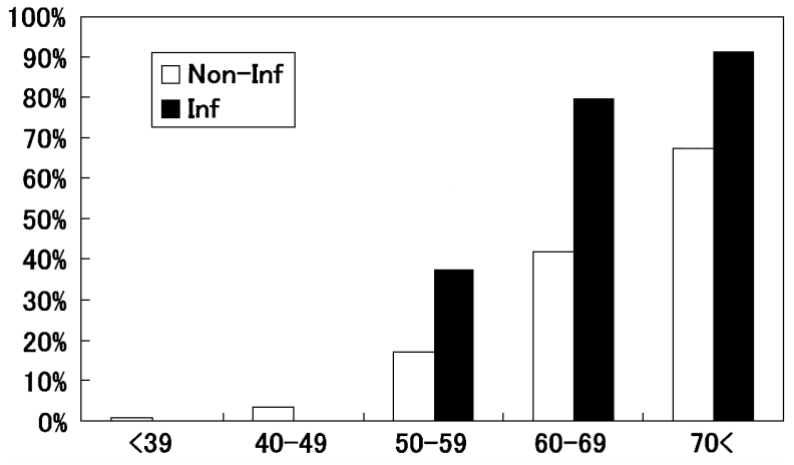

Fig 1. Frequency of aortic calcification according to age groups. Non-cerebral infarction group (Non-Inf, white bars), cerebral infarction group (Inf, black bars).

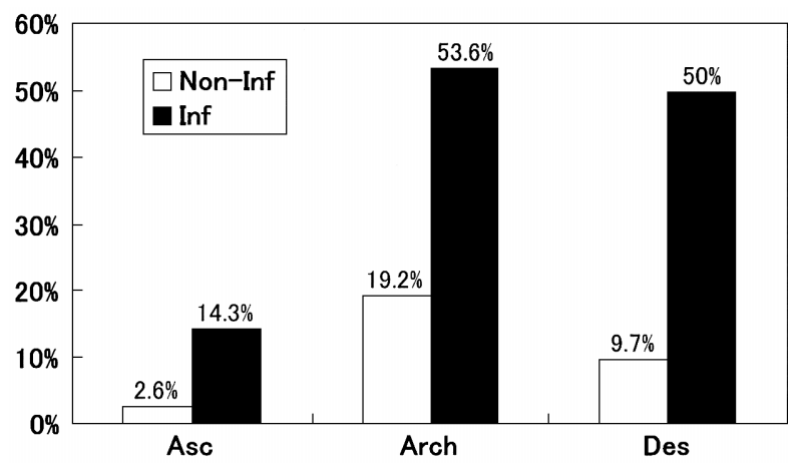

Fig 2. Frequency of aortic calcification at different segments in the overall population. Non-cerebral infarction group (Non-Inf, white bars), cerebral infarction group (Inf, black bars).

ing. The scanning range was from the apex of the lung to the diaphragm and the total scanning time was approximately $30 \mathrm{~s}$, with radiation exposure of $3.6 \mathrm{mSv}$.

All CT data were stored on magnetic-optical discs, and analyzed on a monitor. The window width and level for the CT display were 600 Hounsfield Unit (HU) and $40 \mathrm{HU}$, respectively. In the present study, the software to automatically evaluate calcified lesions was not installed in the monitoring system, so 2 doctors examined all slices twice at separate times for the presence or absence of calcification. The CT density of the aortic wall above $+110 \mathrm{HU}$ was recognized as being AoC. The mean time required for analysis was approximately $2 \mathrm{~min}$. In terms of detecting AoC, there was no discrepancy between either the 2 doctors or the 2 separate examinations.

All participants were asked about risk factors (history of hypertension (HT), hyperlipidemia (HL), diabetes mellitus (DM), smoking and alcohol use) and a past history. Participants who had been medically diagnosed or treated for HT, HL, or DM were considered to have these diseases. Participants who had quit smoking or drinking more than 1 year prior to the screening were included in the category of non-smokers and non-drinkers. Participants who had been hospitalized because of cerebral infarction, intracerebral or subarachinoid hemorrhage (SAH), or definitively diagnosed on a brain $\mathrm{CT}$ or magnetic resonance imaging (MRI) were included in the category of stroke.

We investigated the frequency of $\mathrm{AoC}$ in both the stroke and non-stroke group, and in terms of the contribution of

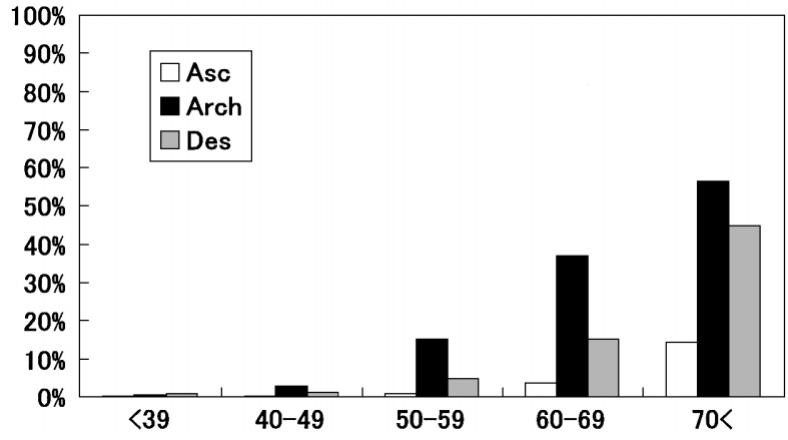

Fig 3. Frequency of aortic calcification at different segments according to age group in the non-cerebral infarction group. Ascending aorta (Asc, white bars), aortic arch (Arch, black bars), descending aorta (Des, gray bars).

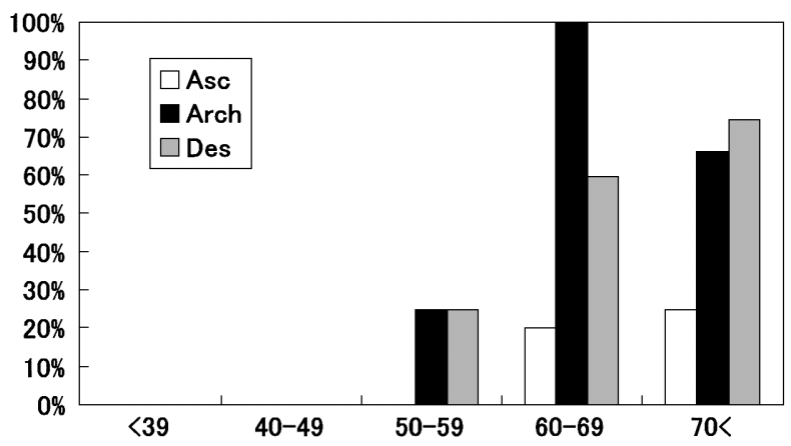

Fig 4. Frequency of aortic calcification at different segments according to age groups in the cerebral infarction group. Ascending aorta (Asc, white bars), aortic arch (Arch, black bars), descending aorta (Des, gray bars).

sex, age, risk factors, and AoC to stroke.

Statistical analyses were performed by using SPSS software (SPSS Japan Inc). Logistic regression analysis was used to evaluate the individual contribution of sex, age, risk factors, and AoC to a past history of stroke. All tests of significance were two-tailed and significance was defined as $\mathrm{p}<0.05$.

\section{Results}

In the present study, 28 subjects (18 men, 10 women; mean age, $65.9 \pm 13.5$ years) had a history of cerebral infarction. None had a history of intracerebral hemorrhage or SAH. Twenty-five of 28 subjects were older than 50 years of age, and 5 subjects had a confirmed diagnosis for cerebral infarction by CT or MRI.

The frequency of $\mathrm{AoC}$ in the overall population was $64.2 \%$ in the infarction group, and $22.1 \%$ in the non-infarction group. This value in the infarction group was significantly higher than that in the non-infarction group who were older than 50 years of age $(\mathrm{p}<0.0001)$ (Fig 1). Fig 2 shows the frequencies of calcification at different segments (ascending aorta (Asc), Arch, and descending aorta (Des)) in the overall population. These values in Asc, Arch, and Des were 14.3, 53.6, and 50\% in the infarction group, and 2.6, 19.2, and $9.6 \%$ in the non-infarction group, respectively. Figs 3 and 4 describe the frequencies of calcification in Asc, Arch, and Des according to age group. These values at different segments in the infarction group were 
also significantly higher than those in the non-infarction group who were older than 50 years of age $(\mathrm{p}<0.05)$.

Table 1 shows the contribution of sex, age, risk factors, and $\mathrm{AoC}$ to cerebral infarction using logistic regression analysis. AoC was a stronger contributor to cerebral infarction than sex, age, and risk factors. In the analysis of the risk estimates of AoC to cerebral infarction as the number of calcified aortic segments increases, the odds ratio of AoC for subjects with a history of cerebral infarction adjusted for sex, age, and risk factors was 1.82 (95\% confidence interval (CI) 1.06-3.15) in men and $2.53(95 \% \mathrm{CI}$ $1.12-5.75)$ in women $(\mathrm{p}<0.05$ for both).

\section{Discussion}

A study using ultrasound images revealed that an increased intima-media thickness (IMT) in the common carotid artery was associated with future cerebrovascular and cardiovascular events ${ }^{26}$ Some studies using transesophageal echocardiography reported that thicknesses of the wall in the Arch, the left common carotid artery, and the left subclavian artery were significantly greater in patients with cerebral infarction than those in the normal population ${ }^{27}$ and that recurrence of ischemic stroke was associated with an increased IMT in the Arch and aortic plaque extension to the branches ${ }^{28}$

Other studies reported that CAC detected by EBCT was associated with a common carotid IMT, ${ }^{29-31}$ and that AoC detected by conventional CT was also associated with the intimal thickness of the thoracic aorta?2 Based on these findings, we hypothesize that calcification in the coronary artery and aorta are associated with an increased incidence and severity of atherosclerotic lesions in the thoracic aorta and the common carotid artery, which are related to the onset of cerebral infarction. The subjects with a history of cerebral infarction showed a higher incidence of $\mathrm{AoC}$ than those without such a history who were older than 50 years of age in the study population. This finding suggests that the incidence of atherosclerotic lesions in the thoracic aorta and the common carotid artery are much higher in those with a history of cerebral infarction who are older than 50 years of age.

In the study by Vliegenthart et al, the odds ratio of CAC for patients with stroke increased with the total calcification score of the coronary artery? ${ }^{4}$ In the present study, the frequency of cerebral infarction was much lower than that in the study by Vliegenthart et al Nevertheless, the odds ratio of AoC for subjects with a past history of cerebral infarction increased as the number of calcified aortic segments increased. This finding suggests that the risk of cerebral infarction can be evaluated by detection of AoC in a mass CT screening. AoC could not be quantitatively evaluated in our previous report because the software to evaluate calcified lesions was not installed in the monitoring system. However, a computer-associated diagnostic system for the evaluation of calcifications in recently improved EBCT and MDCT scanning has been developed. Therefore, the risk of cerebral infarction could be more precisely predicted by evaluating the calcified lesions in the aorta.

With respect to the contribution of sex, age, risk factors, and $\mathrm{AoC}$ to the incidence of cerebral infarction, $\mathrm{AoC}$ was a stronger contributor to that than sex, age and risk factors. However, in our previous study, we also reported that HT and smoking were significantly related to AoC? ${ }^{21}$ Another study suggests that DM and HL are factors that do not
Table 1 Contributions of Aortic Calcification, Sex, Age, and Risk Factors to Cerebral Infarction

\begin{tabular}{cc}
\hline \hline Independent variable & Odds ratio $(95 \% \mathrm{CI})$ \\
\hline AoC & $3.31(1.30,8.47)^{*}$ \\
Age & $1.52(1.01,2.30)^{*}$ \\
Sex & $2.11(0.88,5.10)$ \\
$H T$ & $1.40(0.60,3.25)$ \\
$D M$ & $0.83(0.19,3.62)$ \\
$H L$ & $0.49(0.06,3.69)$ \\
Smoking & $0.72(0.28,1.83)$ \\
Alcohol & $0.84(0.35,2.04)$ \\
\hline
\end{tabular}

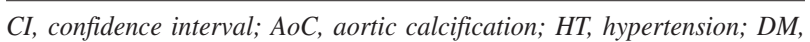
diabetes mellitus; HL, hyperlipidemia. $*_{p}<0.05$.

cause atherosclerosis directly, but rather accelerate the progress of atherosclerosis caused by HT 32 Therefore, when AoC are detected on a CT screening, the presence or absence of risk factors must be carefully evaluated.

In the present study, the relationship between AoC and the incidence of cerebral infarction was investigated. However, there were no patients with a history of intracerebral hemorrhage or $\mathrm{SAH}$, and there was also no information about the long-term prognosis and severity of cerebral infarction in the study population. Therefore, investigations of the influence of AoC on the long-term prognosis, the severity of cerebral infarcton, and the onset of intracerebral hemorrhage or SAH had not been performed previously. Thus, a longitudinal study is needed to confirm the impact of $\mathrm{AoC}$ on these problems.

\section{Conclusion}

The present study results suggest that the detection of aortic calcification in a mass screening for lung cancer and tuberculosis using a mobile helical CT unit is effective to evaluate the risk of cerebral infarction.

\section{References}

1. Masuda Y, Naito S, Takasu J, Watanabe S, Morooka N, Inagaki Y, et al. Coronary artery calcification detected by CT: Clinical significance and angiographic correlates. Angiology 1990; 41: 1037-1047.

2. Masuda Y, Naito S, Aoyagi Y, Watanabe S, Morooka N, Inagaki Y, et al. Clinical importance of coronary calcification detected by CT. Acta Cardiol 1991; 46: 51-56.

3. Naito S, Watanabe S, Masuda Y, Morooka N, Aoyagi Y, Inagaki Y, et al. Evaluation of coronary calcification by computed tomography. J Jpn Coll Angiol 1992; 32: 715-722.

4. Agaston A, Janowitz W, Viamonte M, Gasso J, Kaplan G. Quantification of coronary artery calcium using ultrafast computed tomography. J Am Coll Cardiol 1990; 15: 827-832.

5. Tannenbaum S, Kondos G. Detection of calcific deposits in coronary arteries by ultrafast computed tomography and correlation with angiography. Am J Cardiol 1989; 63: 870-871.

6. Breen J, Sheedy P, Schwarz R, Stanton A, Kaufman R, Moll P. Coronary artery calcification detected with ultrafast $\mathrm{CT}$ as an indication of coronary artery disease. Radiology 1992; 185: 435-439.

7. Shemesh J, Apter S, Rozenman J, Lusky A, Rath S, Motro S. Calcification of coronary arteries: Detection and quantification with doublehelix CT. Radiology 1995; 197: 779-783.

8. Broderick LS, Shemesh J, Wilensky RL, Eckert GJ, Zhou X, Torres WE, et al. Measurement of coronary artery calcium with dual-slice helical CT compared with coronary angiography: Evaluation of CT: Scoring methods, interobserver variations, and reproducibity. AJR Am J Roentgenol 1996; 167: 439-444.

9. Gorich J, Zuna I, Merle M, Beyer-Enke IA, Merkle N, Weigelbaum $\mathrm{K}$, et al. Aortic calcification in CT: Correlation with risk factors and cardiovascular disease. Radiologe 1989; 29: 614-619 (German).

10. Watanabe K, Hiroki T, Koga N. Relation of thoracic aorta calcifica- 
tion on computed tomography and coronary risk factors to obstructive coronary artery disease on angiography. Angiology 2003; 54: $433-441$.

11. Naito S, Watanabe S, Masuda Y, Morooka N, Aoyagi Y, Inagaki Y, et al. Progression to ischemic heart disease in subjects with coronary artery calcification as evaluated by computed tomography. J Cardiol 1990; 20: 249-258.

12. Arad Y, Spadaro L, Goodman K, Newstein D, Guerci A. Prediction of coronary events with electron beam tomography. J Am Coll Cardiol 2000; 36: 1253-1260.

13. Keelan P, Bielak L, Ashai K, Jamjoum L, Denktas A, Rumberger J, et al. Long-term prognostic value of coronary calcification detected by electron-beam computed tomography in patients undergoing coronary angiography. Circulation 2001; 104: 412-417.

14. Raggi P, Cooil B, Callister TQ. Use of electron beam tomography data to develop models for prediction of hard coronary events. Am Heart J 2001; 141: 375-382.

15. Kondos G, Hoff JA, Sevrukov A, Daviglus ML, Garside D, Devries S. Electron-beam tomography coronary artery calcium and cardiac events: A 37-month follow-up of 5635 initially asymptomatic low- to intermediate-risk adults. Circulation 2003; 107: 2571-2576.

16. Sato T, Anno H, Kondo T, Harigaya H, Inoue K, Kakizawa S, et al. Applicability of ECG-gated multislice helical CT to patients with atrial fibrillation. Circ J 2005; 69: $1068-1073$.

17. Kunimasa T, Sato Y, Sugi K, Moroi M. Evaluation by multislice computed tomography of atherosclerotic coronary artery plaques in non-culprit, remote coronary arteries of patients with acute coronary syndrome. Circ J 2005; 69: 1346-1351.

18. Sone S, Takashima S, Li F, Yang Z, Honda T, Maruyama Y, et al. Mass screening for lung cancer with mobile spiral computed tomography scanner. Lancet 1998; 351: 1242-1245.

19. Nakayama T, Kusunoki Y, Suzuki T, Funakoshi T, Imamura F, Horai T, et al. Usage of mobile spiral CT unit into first-step of further examination of lung cancer screening system. J Thorac CT Screen 1998; 5: 104-107.

20. Itani Y, Watanabe S, Masuda Y, Hanamura K, Asakura K, Sone S, et al. Coronary artery calcification detected by a mobile helical CT unit in a mass screening: The frequency and relationship to coronary risk factors and coronary artery disease. Chiba Med J 2001; 77: 123-131.

21. Itani Y, Watanabe S, Masuda Y. Aortic calcification detected in a mass chest screening program using a mobile helical computed tomography unit: The relation to risk factors and coronary artery disease. Circ J 2004; 68: 538-541.

22. Itani Y, Watanabe S, Masuda Y, Hanamura K, Asakura K, Sone S, et al. Measurement of aortic diameters and detection of asymptomatic aortic aneurysm in a mass screening program using a mobile helical computed tomography unit. Heart Vessels 2002; 16: 42-45.

23. Itani Y, Sone S, Nakayama T, Suzuki T, Ito K, Watanabe S, et al. Coronary artery calcification detected by a mobile helical computed tomography unit and future cardiovascular death: 4-year follow-up of 6120 asymptomatic Japanese. Heart Vessels 2004; 19: 161-163.

24. Vliegenthart R, Hollander M, Breteler M, van der Kuip D, Hofman A, Oudkerk M, et al. Stroke is associated with coronary calcification as detected by electron-beam CT: The Rotterdam Coronary Calcification Study. Stroke 2002; 33: 462-465.

25. Iribarren C, Sidney S, Sternfeld B, Browner W. Calcification of the aortic arch risk factors and association with coronary heart disease, stroke, and peripheral vascular disease. JAMA 2000; 283: 28102815 .

26. Bots ML, Hoes AW, Koudstaal PJ, Hofman A, Grobbee DE. Common carotid intima-media thickness and risk of stroke and myocardial infarction: The Rotterdam Study. Circulation 1997; 96: 1432 1437.

27. Nakayama K, Yamamuro A, Ikuno Y, Sakamoto K, Otani S, Hanatani A, et al. Evaluation of patients with cerebral infarction using transesophageal echocardiography: Atherosclerotic changes in the thoracic aorta and the branches of the aortic arch. J Cardiol 1998; 32: $21-30$.

28. Fujimoto S, Yasaka M, Otsubo R, Oe H, Nagatsuka K, Minematsu $\mathrm{K}$. Aortic arch atherosclerotic lesions and the recurrence of ischemic stroke. Stroke 2004; 35: 1426-1429.

29. Oei HH, Vliegenthart R, Hak AE, Iglesias del Sol A, Hofman A, Oudkerk M, et al. The association between coronary calcification assessed by electron beam computed tomography and measures of extracoronary atherosclerosis: The Rotterdam Coronary Calcification Study. J Am Coll Cardiol 2002; 39: 1745-1751.

30. Davis PH, Dawson JD, Mahoney LT, Lauer RM. Increased carotid intimal-medial thickness and coronary calcification are related in young and middle-aged adults: The Muscatine Study. Circulation 1999; 100: 838-842.

31. Wagenknecht LE, Langefeld CD, Carr JJ, Riley W, Freedman BI, Moossavi S, et al. Race-specific relationship between coronary and carotid artery calcification and carotid intimal medial thickness. Stroke 2004; 35: e97-e99.

32. Takasu J. Evaluation of atherosclerotic change of the aorta by enhanced computed tomography. J Jpn Coll Angiol 1990; 30: $1205-$ 1213. 